\title{
Chemical bond overlap properties obtained from localized molecular orbitals and the concept of covalency
}

\author{
Renaldo T. Moura Jr* (PQ) ${ }^{1}$, Albano N. Carneiro Neto (PG) ${ }^{2}$, Oscar M. L. Malta (PQ) ${ }^{2}$ \\ and Ricardo L. Longo (PQ) ${ }^{2}$.renaldo.mourajr@cca.ufpb.br \\ ${ }^{1}$ Department of Chemistry and Physics, Center of Agrarian Sciences, Federal University of Paraíba, \\ Campus II - University City, 58397-000 Areia, PB, Brazil \\ ${ }^{2}$ Department of Fundamental Chemistry, Federal University of Pernambuco, University City, 50670-901 \\ Recife, PE, Brazil
}

Keywords: Chemical bond, overlap, covalency.

\section{Introduction}

The chemical bond overlap properties, originally proposed in 2002 [1], are now formulated on localized molecular orbitals (LMO), which provide the overlap polarizability, intra- and inter-overlap repulsions, and overlap gross population. Thus, it will be presented a development in which the molecular or Kohn-Sham orbitals are calculated and, by a unitary transformation, the localized molecular orbitals (LMOs) are obtained. Subsequently, the atomic (one center) and overlap (two centers) contributions to the chemical bond properties are evaluated. This new approach was applied to a large set of systems ranging from simple diatomic and polyatomic molecules. The overlap properties revealed to be closely related with the Charge Shift Resonance Energies and to bond critical points, indicating that the lone pair bond weakening effect and the presence of compact orbitals affect the trends observed for the overlap properties and can be quantified by our proposed model.

\section{Methodology}

Given the localized character of the chemical bond overlap, is reasonable to use LMO to obtain its properties. The total electron density can be decomposed to provide the overlap density according to Eq. (1). The overlap contributions for the gross population can be obtained by numerical integration over the population density $\rho_{\mathrm{OP}}(r)$ in Eq. (1) or by analytical population analysis

$$
\begin{gathered}
\rho_{\mathrm{OP}}(r)=\sum_{i \in A}^{m} \sum_{j \in B}^{m} c_{i} c_{j} \varphi_{i} \varphi_{j} \\
p_{\mathrm{OP}}=4 \sum_{i \in A}^{m} \sum_{j \in B}^{m} c_{i} c_{j} S_{i j}
\end{gathered}
$$

obtained by the Eq. (2), where $S_{i j}$ are the overlap integrals on the atomic basis functions that can be obtained analytically and $c_{i} c_{j}$ are generally called density matrix elements and can be obtained from a localization procedure for the molecular system. The repulsion between two overlap electron densities can be calculated as:

$$
\mathrm{J}_{\mathrm{OP}}^{\text {intra }}=\sum_{x i} \sum_{y i}^{M 1} \sum_{z i} \sum_{x j} \sum_{y j}^{M 2} \sum_{z j} \rho_{\mathrm{OP}}\left(r_{i}\right) \cdot r_{12}^{-1} \cdot \rho_{\mathrm{OP}}\left(r_{j}\right) \Delta v_{1} \Delta v_{2}
$$

where $\rho_{\mathrm{OP}}\left(r_{i}\right)$ and $\rho_{\mathrm{OP}}\left(r_{j}\right)$ are points in each of the overlap densities and $\Delta v$ are the volume elements. The summations run over mesh points in the electronic population density and can be solved using an adaptive Monte Carlo method, where the space discretization is performed adaptively in an iterative process. The chemical bond overlap polarizability can be calculated as follows: 


$$
\alpha_{\mathrm{OP}}^{\kappa \lambda}=-\frac{2}{F_{\kappa}}\left(\sum_{\substack{i \in A \\ i \in B}}^{m} \sum_{\substack{j \in B \\ j \in A}}^{n} c_{i}^{\prime} c_{j}^{\prime}\left\langle\varphi_{i}|\lambda| \varphi_{j}\right\rangle-\sum_{\substack{i \in A \\ i \in B}}^{m} \sum_{\substack{j \in B \\ j \in A}}^{n} c_{i}^{0} c_{j}^{0}\left\langle\varphi_{i}|\lambda| \varphi_{j}\right\rangle\right), \quad \kappa, \lambda=x, y, z
$$

where $c_{i}^{0}$ and $c_{i}^{\prime}$ are the expansion coefficients for the unperturbed and perturbed LMO. These coefficients can be easily obtained from a self-consistent field (SCF) procedure followed by a localization unitary transformation.

The calculations of these overlap properties are implemented in our software Bond Overlap Polarizability Package (BOPP), that works together with the GAMESS program and was applied to a large set of diatomic and polyatomic molecules. The geometry optimization and localization (with Pipek-Mezey method) calculations were performed with the B3LYP functional using the aug-cc-pVTZ basis set.

\section{Results and discussion}

For the B-H, C-H, N-H, O-H and S-H chemical bonds in $\mathrm{BH}_{3}, \mathrm{CH}_{4}, \mathrm{NH}_{3}, \mathrm{H}_{2} \mathrm{O}$ and $\mathrm{H}_{2} \mathrm{~S}$ molecules (entries a-e in Fig. 1), there is a trend in the variation of $\alpha_{\mathrm{OP}}, p_{\mathrm{OP}}$, and $\mathrm{J}_{\mathrm{OP}}^{\text {intra }}$ values. It is noted that $\alpha_{\mathrm{OP}}$ decreases in the order $\mathrm{B}-\mathrm{H}>\mathrm{C}-\mathrm{H}>\mathrm{N}-\mathrm{H}>\mathrm{O}-\mathrm{H}$, indicating that the increase of lone pairs presence in the carbon atom (without lone pairs), nitrogen atom (one lone pair) and oxygen atom (two lone pairs) can promote an increase in the repulsion between the lone pair and the chemical bond overlap, thus decreasing the $\mathrm{J}_{\mathrm{OP}}^{\text {intra }}$ and $p_{\mathrm{OP}}$ values. This trend also follows the variation of the electronegativity of the heteroatom. The $\mathrm{S}-\mathrm{H}$ bond on $\mathrm{H}_{2} \mathrm{~S}$ molecule shows a larger overlap polarizability than the $\mathrm{O}-$ $\mathrm{H}$ bond in $\mathrm{H}_{2} \mathrm{O}$.

The Atom-in-Molecule (AIM) properties calculated for these molecules show a straightforward correlation with the overlap polarizability properties. Because the value of $-\nabla^{2} \rho$ at a point quantifies how the electronic charge concentrates around that point, then larger values of $-\nabla^{2} \rho$ or, more precisely, the more negative values of $\lambda_{1}$ and $\lambda_{2}$, more polarizable would be the bond overlap density. This trend can be observed in the results, indicating the robustness of the Chemical Bond Overlap Model, that is consistent with a

Figure 1. Overlap population density in polyatomic molecules color-mapped with isovalues in $e / a_{0}^{3}$.

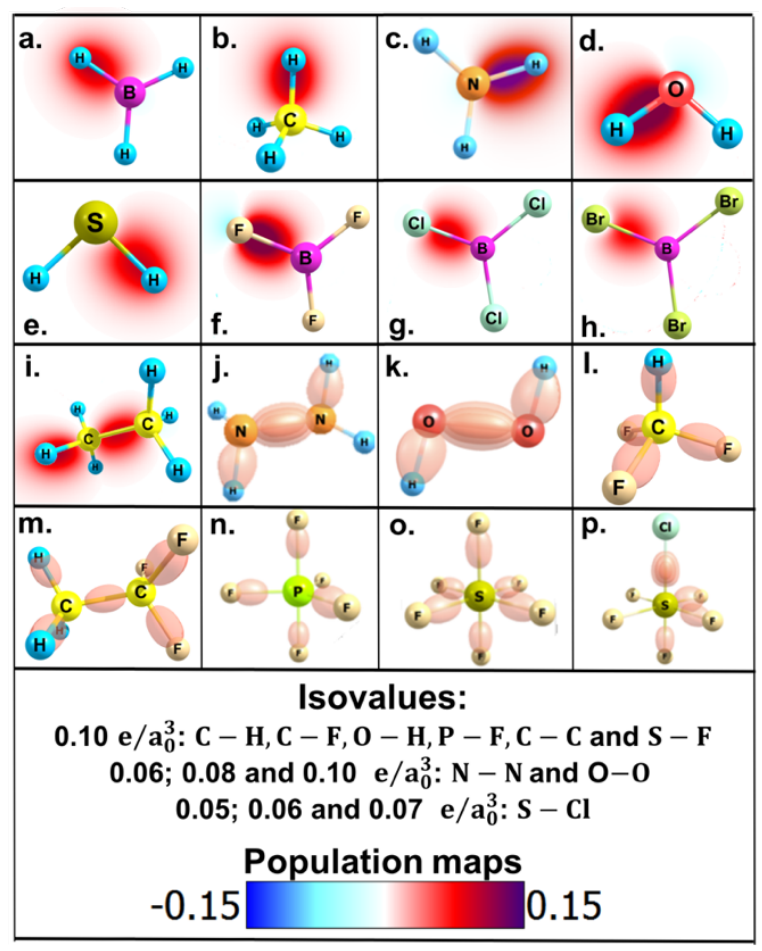
well-established chemical bond analyses such as AIM as well as the charge shift resonance energies, which shall be discussed in details during the presentation.

\section{Acknowledgments:}

\section{References}

We thank FACEPE, CAPES and CNPq for their support.

[1] O.L. Malta, H.J. Batista, L.D. Carlos, Chem. Phys. 21282 (2002). 